\title{
Article \\ A Position Sensing Glove To Aid Ankle-Foot Orthosis Diagnosis and Treatment
}

\author{
Eduard Cazacu ${ }^{1}$, Coen van der Grinten ${ }^{1}$, Jeroen Bax ${ }^{1}$, Guus Baeten ${ }^{1}$, Fred Holtkamp ${ }^{2}$, and Chris Lee ${ }^{1, *} \mathbb{D}$ \\ 1 Fontys Institute of Engineering, Fontys University of Applied Sciences \\ 2 Fontys school for Allied Health Professions, Fontys University of Applied Sciences; f.holtkamp@fontys.nl \\ * Correspondence: c.lee@fontys.nl
}

\begin{abstract}
A position sensing glove, called SmartScan, that creates a 3D virtual model of a real object is presented. The data from the glove is processed by a volume minimization algorithm to validate the position sensor data. This allows only data from the object's surface to be retained. The data validation algorithm allows the user to progressively improve an image by repeatedly moving their hand over the object. In addition, the user can choose their own balance between feature resolution and invalid data rejection. The SmartScan glove is tested on a foot model and is shown to be robust against motion artifacts, and has a mean accuracy of $2.9 \mathrm{~mm}$ (compared to a 3D model generated from optical imaging) without calibration.
\end{abstract}

Keywords: ankle-foot orthosis; position sensors; smart glove; 3D modeling

\section{Introduction}

It has been estimated that about $57 \%$ of the Dutch population will suffer from foot problems at some point in their lives [1]. This figure is broadly representative of western European nations [2]. Magnusson et al. report that about 2.2\% of the population of India have some kind of physical disability [3], including ankle-foot orthoses. Despite the relative prevalence of ankle-foot orthosis, successful treatment is inefficient, with about $20 \%$ of corrective measures failing to reduce suffering [4].

The high failure rate of ankle-foot orthosis is not due to the lack of skill or training, but rather, the lack of quantitative data [5]. The current standard involves the clinician taking a (plaster) cast of the foot while holding it in the corrected position. The clinician has no quantitative data about the correction, and must rely on experience to obtain an accurate cast of the corrected foot. In addition to the lack of data, the process is wasteful —each foot correction requires a plaster cast—and slow, as the cast must be shipped to another location for processing.

To turn ankle-foot orthosis into a data-driven process, we propose the creation of virtual foot models that can be manipulated by the clinician in order to better control and test different possible corrections. A virtual model of a foot can be easily created using laser scanning technology [6]. Laser scanning is highly accurate and rapid. The rapidity of laser scanning eliminates motion artifacts. Unfortunately, if the clinician manipulates the foot (an integral part of diagnosis) during imaging, the manipulation is obscured by the clinician's hands. Thus, it becomes difficult to extract a virtual model of the manipulated foot. Roberts et al. reported that an increased delay between the initial scan measurements and successful treatment [6].

It is now possible to use a phone camera or table camera to create a 3D model of a foot. However, Farhan et al. found that the quality of evidence that 3D scanning techniques, including laser scanning, improve healthcare outcomes is weak [7]. Although the reasons for the lack of improvement do not appear to be well investigated, some possible explanations include distortions due to motion artifacts, and that the images include the hands of the clinician, obscuring the manipulated foot, and preventing the creation of an accurate determination of the required mould. 
Here, we propose SmartScan, a glove that allows the clinician to create a 3D virtual model of the foot by feeling and manipulating the foot. The use of smart gloves in virtual reality is common. Gloves with pressure sensors and accelerometers are commonly used to translate real-world object manipulation into virtual reality [8]. However, the object to be manipulated is often pre-programmed into the environment.

Similarly, smart gloves can be used to virtually sculpt objects [9-11]. In these cases, a virtual object is manipulated using a smart glove that has position sensors and/or accelerometers. Unlike the virtual reality model above, no real object exists and motion is translated purely into the manipulation of a virtual object.

To our knowledge, there is no system that generates a virtual object from the motion of a smart glove around a real object. For the diagnosis and treatment of ankle-foot orthosis, such a glove may be a significant step in improving treatment success rates. A smart glove can be used to recreate a model of the foot, including the way that the clinician moulds the shape of the foot. The clinician can then use virtual reality to manipulate the foot model and judge which shape is most appropriate. From there, a CAD model of the required mould can be created and manufactured using 3D printing, speeding up treatment and reducing waste.

In this paper, we present a proof-of-concept of SmartScan, a glove fitted with position sensors. We present the data processing algorithm that is used to validate the position data and generate an accurate 3D model of an object that is manipulated by the user. We find that the accuracy of the SmartScan point cloud is comparable to that of an optical scanner.

\section{Materials and Methods}

\subsection{Hardware}

Position sensors (NDI 3D Guidance model 180) with six degrees of freedom are mounted on a glove. The sensors use an electromagnetic field reference system (by measuring phase, amplitude and polarization relative to a reference, the three dimensional location and orientation of the sensor can be calculated relative to the reference location). The commercial sensor system is capable measuring locations within a volume of 75 $\mathrm{cm}$ per side with a position accuracy of $1.4 \mathrm{~mm}$ (RMS) and an angular accuracy of $0.5^{\circ}$ (RMS) and at a sample rate of up to $2 \mathrm{kHz}$. Although the system is capable of sampling up to 25 position sensors, for the prototype, three sensors are mounted on the glove (one on the thumb, one on the forefinger and one on the middle finger). A fourth sensor is mounted on the object of interest to counteract motion artifacts.

In using SmartScan, the user is required to provide the system with at least one reference point. Thus, before "scanning" the object, the user first uses the glove to define a series of reference points (see Sec. 2.2) and then begins the scan.

\subsection{Software}

The sensor data is transmitted to a laptop where a service (see Fig. 1(a) and Tab. 1) receives and processes the data. The data processing pipeline, shown in Fig. 1(b), consists of two threads: one dedicated to receiving and storing the data in memory, while the other is devoted to filtering. The filtered data is then either transferred to the CAD software, or is stored to disk in a comma separated value file. The service is implemented so that it can run as a standalone application or as a plugin in the CAD software system.

The position data from the sensor is translated and rotated to the reference frame of the reference sensor. In this way, if the object of interest moves during the scan, the image is not distorted.

When using the software, the user is asked to define reference points, which is the average location of all the sensors (excluding the reference sensor) for a fixed period of time. The user may define as many reference points as required. The role and requirements of the reference points is explained more fully in Sec. 2.3. 


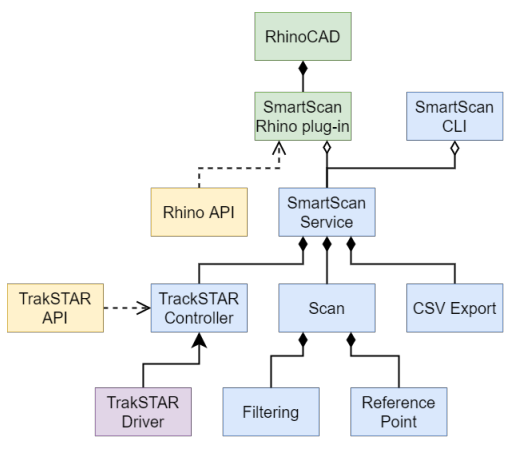

(a)



(b)

Figure 1. Software Architecture (a). Green: RhinoCAD, SmartScan Rhino Plug-in, Blue: SmartScanService, TrakSTAR Controller, Scan, CSVExport, Filtering, ReferencePoint, Yellow: RhinoAPI, TrakSTAR API, purple: TrakSTAR driver. Data processing pipeline (b). Yellow: acquisition thread, Green: filtering thread

\begin{tabular}{|l|l|}
\hline Block & Description \\
\hline RhinoCAD & CAD software \\
\hline SmartScan Rhino plug-in & $\begin{array}{l}\text { RhinoCAD plug-in for measurement control and } \\
\text { visualization }\end{array}$ \\
\hline Rhino API & $\begin{array}{l}\text { Software library used to interface with the } \\
\text { RhinoCAD application. }\end{array}$ \\
\hline SmartScan CLI & Command line interface for the Smart-Scan system. \\
\hline SmartScanService & $\begin{array}{l}\text { The Smart-Scan software library. } \\
\text { Implemented as a C++ class. }\end{array}$ \\
\hline TrakSTAR Controller & Class that interfaces with the TrakSTAR driver \\
\hline Scan & $\begin{array}{l}\text { Class encapsulating the measurement } \\
\text { and filtering pipeline. }\end{array}$ \\
\hline Filtering & Class implementing the filtering algorithms \\
\hline ReferencePoint & Custom datatype for reference points. \\
\hline CSV Export & Class for formatting and exporting the data \\
\hline TrakSTAR API & $\begin{array}{l}\text { Software library facilitating TrakSTAR driver } \\
\text { communication }\end{array}$ \\
\hline TrakSTAR Driver & Microsoft Windows driver for the TrakSTAR device \\
\hline
\end{tabular}

Table 1. Software blocks for sensor data collection, filtering, and visualization.

\subsection{Filter algorithm}

Although the position sensors are highly accurate, SmartScan does not have any intrinsic knowledge of its environment. The data from the sensors report the position irrespective of whether the user's fingers are touching the object of interest or not. Therefore, it is necessary to select the data that corresponds to when the user is touching the object of interest. Furthermore, because it is desirable that the clinician not be required to change their behavior, the selection algorithm should operate with minimal user interaction.

Filtering is accomplished in two steps. The first step is a volume minimization filter, which, based on the location of a reference point, selects only those points that lie on a surface that encloses the reference location in the smallest volume. The second step searches for, and removes, outliers that the first filtering step misses.

Volume minimization is illustrated in Fig. 2. In short, SmartScan is used to scan a solid object, therefore, it is expected that there is a volume in which there are no position data points. Through a sensible choice of glove position, the user can ensure that the reference data points are located within the object's volume. 


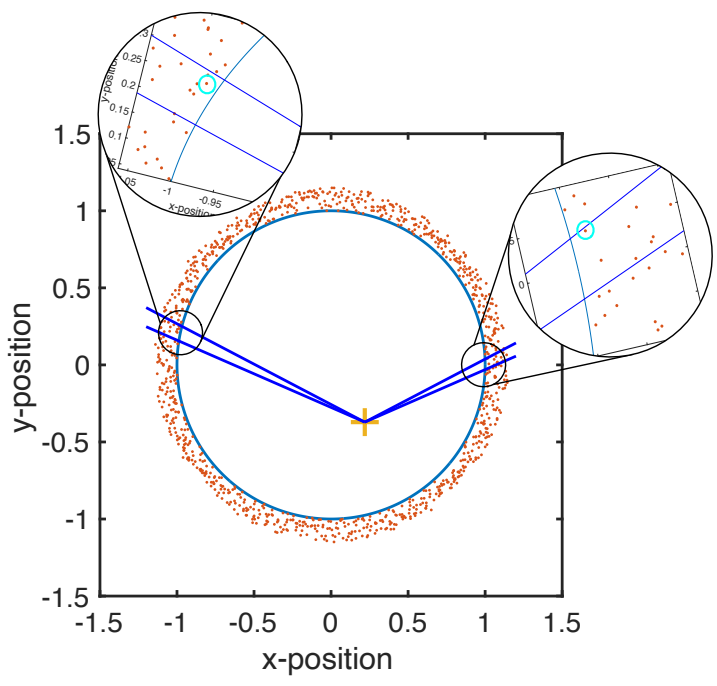

(a)



(b)

Figure 2. Single reference point in a spherical volume (a). Within the angular resolution (dark blue lines), only the point closest to the reference point (highlighted by the cyan circles in the insets) are passed on for visualization. With a near spherical object, the areal density of points after filtering is nearly constant. Single reference point in a cylindrical volume (b). As with the case for the spherical volume, only the closest point to the reference point is selected. However, for some locations, the areal density of points is significantly reduced. In addition, due to the highly oblique angle, the selected point is not necessarily close to the surface (cyan circle top inset).

As shown in Fig. 2, the reference point acts as an origin, and the volume is divided into solid angular sections with a user specified angular size. Within each section, only the points closest to the origin are selected and the rest are discarded. Fig. 2a shows that for a spherical (or near spherical object), a single well-chosen reference point is sufficient to select the sensor positions that correspond to the surface. Furthermore, the spacing between the selected positions will remain relatively constant (assuming a sufficient density of initial data).

However, Fig. 2(b) shows that, for non-spherical objects, a single reference point results in increasingly large spacing between positions. This is because the vector between the origin and the surface rotates from near normal to highly oblique. At an oblique angle, a single data point will represent a large surface area, resulting in a low resolution model.

To maintain a nearly constant lateral resolution, more reference points should be used. Each reference point is used to validate the data that is closest to it. The user must select enough reference points to ensure that the vector between the object surface and the closest reference point does not become highly oblique.

A weakness of the filtering methodology described above is that if there is only a single data within an angular section, then it is automatically chosen. This can result in outliers that distort the final image (see Fig. 3). To eliminate the outliers, a modified version of the same volume minimization algorithm is used. Instead of selecting only the closest points, the algorithm eliminates points that are more than $20 \%$ further away than the average of the data within that angular section. The outlier filter operates after the selection filter and at a coarser angular resolution than the selection filter so that sufficient data are used to calculate the average.

Note, as shown in Fig. 1(b), the pipeline only passes validated data for visualization. However, the selection process always operates on the entire data set. In other words, 


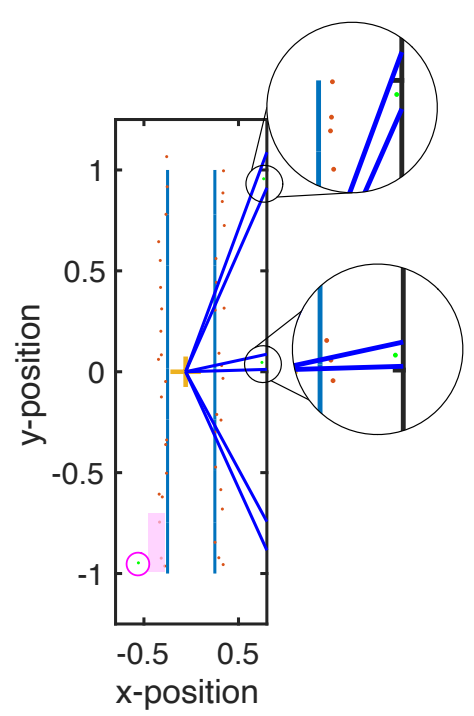

Figure 3. In irregular geometries or when the data density is low, outliers can be selected as valid, as shown in the top inset. The lower inset shows a case where an outlier is rejected. To prevent outliers from being selected, a coarser angular range is used to select points for which the mean distance to the reference is calculated (purple block). The mean is used to exclude points that are more than $20 \%$ greater than the mean (magenta circle).

if a user scans their hand over one section of an object and then later returns to that section, both the old and the newly acquired data points are included in the filtering process. Since the CAD software renders the point cloud in near real-time, the user is able to observe the build up of the model and can rescan areas where the data density is either too low, or has many outliers.

\section{Results}

The selectivity of the sensor was tested on a cylinder (approximately $7 \mathrm{~cm}$ in diameter and $20 \mathrm{~cm}$ high). As can be seen in Fig. 4, the percentage of retained data drops sharply with coarser angular resolution.

It is important to note that the data selection process is a balance between three conflicting requirements. High spatial resolution images require that the angular resolution of the filter is maintained at a high value. However, if the angular resolution is too high, then the selectivity of the algorithm drops and the resulting virtual image is noisy. Furthermore, the time it takes for the filter to process the data increases, making it difficult to maintain real-time visualization. On the other hand, if the angular resolution is decreased, the filter operates faster, and decreases the noise in the virtual image. However, the resolution of small features is compromised by the relatively poor angular resolution.

The quality of the resulting image was compared by scanning a model foot and applying different filter settings to the saved data. The result of this can been in Fig. 5 . For these data, two reference locations were set: one near the bridge of the foot and one near the ankle.

The improvement of the 3D model clarity over time is shown in Fig. 6 for a resolution of $1^{\circ}$. The sub-figures (A)-(C) show the point cloud as the scan proceeds in time. The user is repeatedly moving their hand over the foot, adding new points for the selection algorithm to validate. As a result, outliers and points that are not on the surface are progressively removed.

A scan of a model foot (angular resolution of $4^{\circ}$, resulting in 2874 valid points) is compared with a standard optical imaging technique (Occipital structure scanner, connected to an iPad) in Fig. 7. It is clear that the resulting scan is reasonably close to that obtained by the optical imaging technique. It is notable that the data selection algorithm 


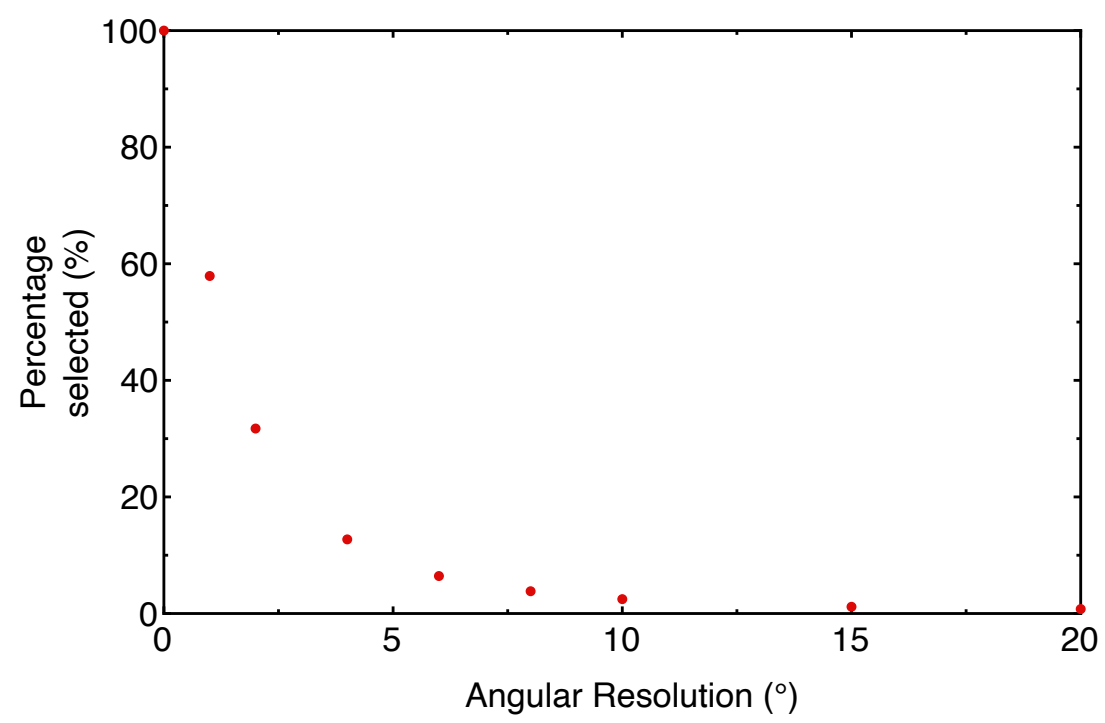

Figure 4. The selectivity of the filtering algorithm for increasing angular resolution. As can be seen the percentage of retained data falls off sharply with coarser angular resolution.
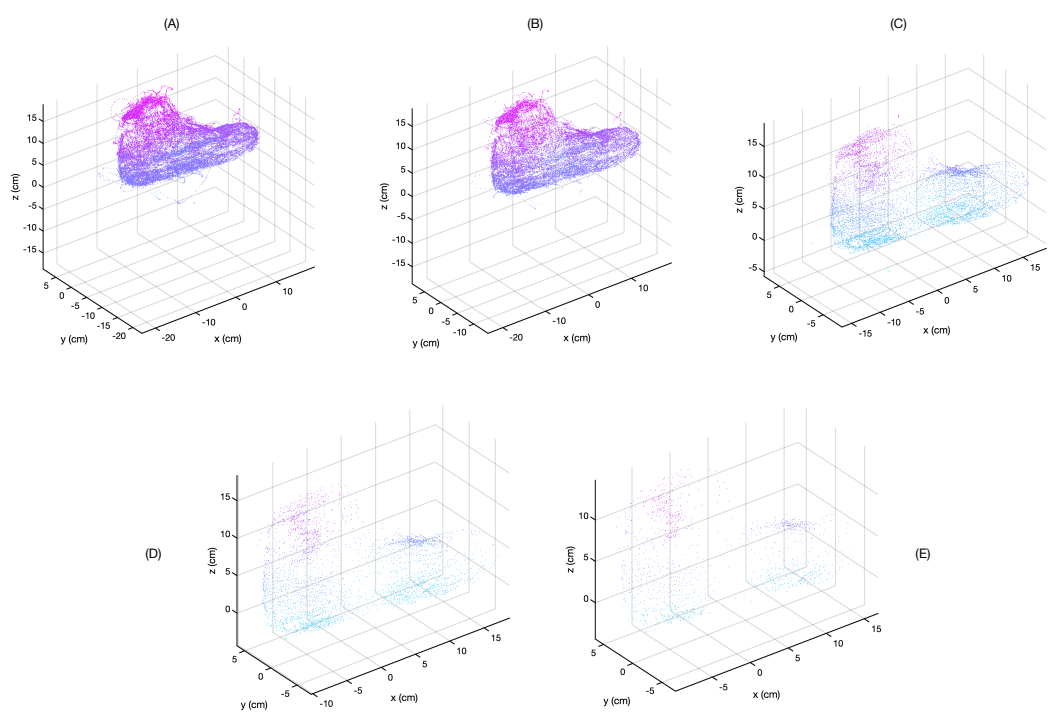

Figure 5. The effect of filtering. As the angular resolution of the filter decreases, the number and extremity of the outliers decreases. However, the total number of data points decreases sharply and the image resolution becomes poorer. The resolutions are, in order from (A)-(E): $0.25^{\circ}, 1^{\circ}, 3^{\circ}$, $5^{\circ}$, and $8^{\circ}$.
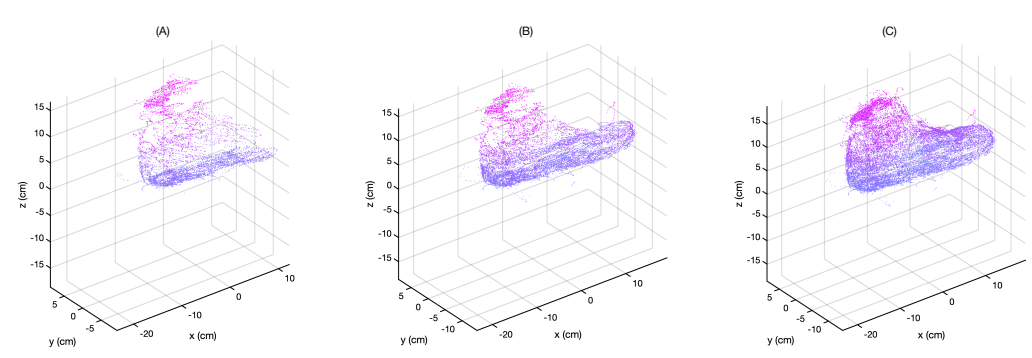

Figure 6. Build up of visualization as the number of points increases from 0 to 10,000 (A), to 20,000 (B), and to 30,000 (C) at an angular resolution of $1^{\circ}$. The removal of points that do not lie on the surface is particularly notable on the bridge of the foot. In (A), the bridge consists of points that do not lie on the surface, while in (B) and (C) the bridge surface is better defined. 


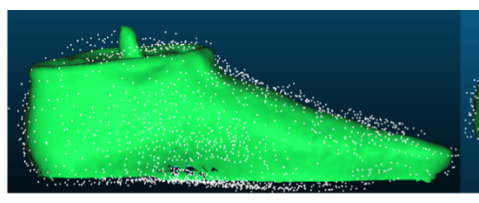

(a)

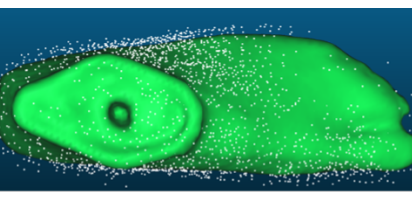

(b)

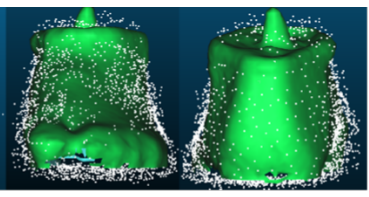

(c)

Figure 7. Comparison between optical imaging (green image) and scan data (point cloud). The two techniques agree within $3 \mathrm{~mm}$ in general with some outliers still selected as valid data.

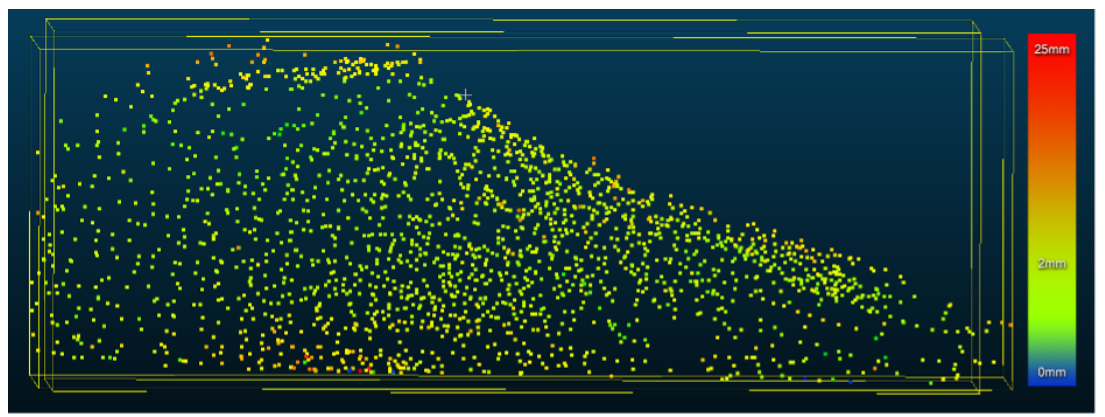

Figure 8. Point cloud of model foot taken with $4^{\circ}$ resolution. The color of the points indicates the distance between the point and the nearest mesh surface from an optical image of model foot (not shown for clarity). The average is $2.9 \mathrm{~mm}$, but some outliers $(\sim 25 \mathrm{~mm})$ are still taken as valid data.

removes the vast majority of invalid data points, but is not perfect. For example, the accumulation of data on the side of the foot in Fig. 7(c) reduces the image accuracy.

CloudCompare [12] was used to measure the distance between the mesh generated by the optical scan and the point cloud generated from the glove (see Fig. 8). The average separation was found to be $2.9 \mathrm{~mm}$. However, outliers, with a distance up to $25 \mathrm{~mm}$ are also present.

\section{Discussion}

The smart glove presented here represents the beginning a new concept in the treatment of ankle-foot orthosis. The glove allows a 3D model of the foot to be built up while it is being manipulated by the clinician. By using a minimum volume algorithm to validate the data, the resulting model is within $3 \mathrm{~mm}$ of more commonly used optical imaging models. However, this is still large compared to the estimated accuracy of plaster casts $(\sim 1 \mathrm{~mm})$ that are currently used.

A portion of the error is due to the placement of the sensor and the lack of calibration. The sensor is placed on the glove and is not always in contact with the foot model, even when the fingers are in contact with the model. It is, therefore, important to add a calibration step to the software.

Nevertheless, even in its current form, the sensing glove is clinically useful when paired with an optical imaging technique. The smart glove data is validated by minimizing the distance to a reference point, and, thereby the volume of the shape. Many manipulations involves pressing the foot into a new shape locally, which reduces the distance to the reference point (and effectively reduces the volume ). Thus, the resulting 3D model includes these manipulations by default. By comparing the optically obtained 3D model with the model obtained from the smart glove, the shape obtained from the clinician's foot manipulation can be extracted from the difference between the two 3D images.

Nevertheless, it is also desirable to reduce the number of data points that are invalid, and to separate out the natural shape of the foot from the desired shape of the foot. To achieve these goals, SmartScan will be improved by including pressure sensors and machine learning. In addition to improved data processing, these additional steps will 
(a) help clinicians improve their practice and to provide a more robust data validation algorithm. Machine learning algorithms may also provide an additional data validation step. Machine learning may also help separate out areas of interest for the clinician by examining aspects such as the time spent in a particular location, or how often the clinician returns to a location.

Author Contributions: “Conceptualization, C.L. and F.H..; methodology, C.L., C.G, and J. B..; software, E. C., M. K., A. S.; testing, G. B. and A. C; supervision, C. L.; project administration, F. H.; funding acquisition, F. H. All authors have read and agreed to the published version of the manuscript.

Funding: This research was funded by SIA-RaaK grant number RAAK.MKB12.032.

Institutional Review Board Statement: Not applicable

Informed Consent Statement: Not applicable

Data Availability Statement: Code and data is available at https:/ / github.com/exMamaku/smartscan

Conflicts of Interest: The authors declare no conflict of interest.

\section{References}

1. Aantal gebruikers 2016-2020, per hulpmiddelencategorie. Available online: https:/ /www.gipdatabank.nl/ (accessed on 09-072021).

2. Burzykowski, T.; Molenberghs, G.; Abeck, D.; Haneke, E.; Hay, R.; Katsambas, A.; Roseeuw, D.; Van De Kerkhof, P.; Van Aelst, R.; Marynissen, G. High prevalence of foot diseases in Europe: results of the Achilles Project. Mycoses 2003, 46, 496-505, [https://onlinelibrary.wiley.com/doi/pdf/10.1046/j.0933-7407.2003.00933.x]. doi:10.1046/j.0933-7407.2003.00933.x.

3. Magnusson, L.; Ghosh, R.; Jensen, K.R.; Göbel, K.; Wågberg, J.; Wallén, S.; Svensson, A.; Stavenheim, R.; Ahlström, G. Quality of life of prosthetic and orthotic users in South India: a cross-sectional study. Health and Quality of Life Outcomes 2019, 17, 50. doi:10.1186/s12955-019-1116-y.

4. Holtkamp, F.C.; Wouters, E.J.M.; van Hoof, J.; van Zaalen, Y.; Verkerk, M.J. Use of and Satisfaction with Ankle Foot Orthoses. Clinical Research on Foot $\mathcal{E}$ Ankle 2015, 3, 167.

5. Holtkamp, F.C.; Verkerk, M.J.; van Hoof, J.; Wouters, E.J.M. Mapping user activities and user environments during the client intake and examination phase: An exploratory study from the perspective of ankle foot orthosis users. Technology and Disability 2016, 28, 145-157. doi:10.3233/TAD-160452.

6. Roberts, A.; Wales, J.; Smith, H.; Sampson, C.J.; Jones, P.; James, M. A randomised controlled trial of laser scanning and casting for the construction of ankle-foot orthoses. Prosthet Orthot Int 2016, 40, 253-261. doi:10.1177/0309364614550263.

7. Farhan, M.; Wang, J.Z.; Bray, P.; Burns, J.; Cheng, T.L. Comparison of 3D scanning versus traditional methods of capturing foot and ankle morphology for the fabrication of orthoses: a systematic review. J Foot Ankle Res 2021, 14, 2. doi:10.1186/s13047-020-00442-8.

8. Dipietro, L.; Sabatini, A.M.; Dario, P. A Survey of Glove-Based Systems and Their Applications. IEEE Transactions on Systems, Man, and Cybernetics, Part C (Applications and Reviews) 2008, 38, 461-482. doi:10.1109/TSMCC.2008.923862.

9. Han, Y.C.; Han, B.j. Virtual pottery: a virtual 3D audiovisual interface using natural hand motions. Multimedia Tools and Applications 2014, 73, 917-933. doi:10.1007/s11042-013-1382-3.

10. Kiliboz, N.Ç. Virtual Sculpting With Advanced Gestural Interface. Master's thesis, Bilkent University, 2013.

11. Kao, C.H.; Chen, C.C.; Jhu, W.Y.; Tsai, Y.T.; Chen, S.H.; Hsu, C.M.; Chen, C.Y. Novel digital glove design for virtual reality applications. Microsystem Technologies 2018, 24, 4247-4266. doi:10.1007/s00542-018-3747-z.

12. CloudCompare. Available online: https://www.danielgm.net/cc/ (accessed on 09-07-2021). 